\title{
Heavy Users: Mysterious Research
}

Das Besondere an der Heavy-user-Forschung ist, dass sie sich seit über 20 Jahren mit Engagement einer Patientengruppe widmet, für die es keine einheitliche Bezeichnung gibt, die nach wie vor einer klaren Definition entbehrt und deren tatsächliche Existenz immer noch nicht zweifelsfrei feststeht.

Personen, die medizinische Leistungen stark in Anspruch nehmen, werden in der Versorgungsforschung wahlweise als „,heavy user“, „high utilizer“, „frequent repeater“ oder „multiple recidivists“ bezeichnet, aber auch die Kombinationen „high user“, „heavy utilizer“ und „frequent user“ sind möglich. Dies ist ein Bonus für die sprachliche Vielfalt, aber nicht für die inhaltliche Präzision. Die babylonische Sprachverwirrung macht Literaturrecherchen zu einer Sisyphosarbeit und lässt die Wahrscheinlichkeit, einen vollständigen Überblick über den Stand der Forschung zu gewinnen, drastisch sinken.

Patienten, die bestimmte medizinische Versorgungsangebote überdurchschnittlich häufig nutzen, gibt es in allen Bereichen der Gesundheitsversorgung, vom Krisentelefon bis zum Krankenhaus. Da die stationäre Versorgung jedoch sehr kostenintensiv ist, hat eine überproportionale Inanspruchnahme in diesem Bereich besondere Bedeutung und bildet folgerichtig den Fokus der Heavy-user-Forschung.

Während schon seit den 70er Jahren Untersuchungen zu den Ursachen und Prädiktoren stationärer Wiederaufnahmen durchgeführt wurden, sind heavy user stationärer Behandlung erst seit den 80er Jahren ein Schwerpunkt der psychiatrischen Versorgungsforschung. Das besondere Interesse an heavy usern erklärt sich dadurch, dass vermutet wird, die starke Leistungsinanspruchnahme dieser Patientengruppe sei unter bestimmten Bedingungen vermeidbar. Die Versorgungsforschung versucht deshalb, die Ursachen intensiver Inanspruchnahme zu identifizieren sowie geeignete alternative Therapieangebote und Interventionsmöglichkeiten abzuleiten.

Dieses Forschungsziel macht eine Differenzierung zwischen „starker“ und „normaler“ Nutzung medizinischer Versorgung notwendig. Die meisten Studien orientieren sich dazu an der Häufigkeit der Patientenkontakte mit bestimmten Einrichtungen, finden aber keine einheitliche Definition dafür, wie viele Kontakte im Beobachtungszeitraum erfolgen müssen, damit von starker Inanspruchnahme gesprochen werden kann. Dies ist nicht verwunderlich, da nur schwer zu begründen ist, warum beispielsweise drei stationäre Aufnahmen pro Jahr eine „starke“ Nutzung darstellen sollen, während zwei Krankenhausaufenthalte in dieser Zeit noch „normal“ sind. Das bedeutet: Bislang ist es nicht gelungen, einen Punkt zu finden, in dem sich Patienten mit einer stärkeren Inanspruchnahme eindeutig von Patienten mit einer geringeren Inanspruchnahme unterscheiden. Wenn man bei dem gewählten Beispiel bleibt, gilt das, was für „heavy user“ mit mindestens drei Aufnahmen herausgefunden wurde, in abgeschwächter Form auch noch für „Normalnutzer“ mit zwei Aufnahmen. Heavy user und Normalnutzer können demnach bislang nur quantitativ, aber nicht qualitativ voneinander abgegrenzt werden. Die Entscheidung, ab wann von heavy use gesprochen wird, ist daher willkürlich und bleibt letztlich den einzelnen Autoren überlassen.

Aufgrund der genannten definitorischen Probleme, aber auch infolge unbefriedigender Studienergebnisse, bezweifeln einige Autoren sogar, dass es heavy user als eigenständige Patientengruppe mit spezifischen Merkmalen überhaupt gibt [1]. Dagegen sind andere Forscher so überzeugt von der Existenz der heavy user, dass sie bereits detaillierte Subgruppenprofile erarbeitet haben [2-4]. 
In Anbetracht der beschriebenen Verwirrung verwundert es nicht, dass, wenn es um heavy user geht, immer wieder der Begriff „Phänomen“ auftaucht. Eine Bezeichnung, die einen ebenso mystischen wie unwissenschaftlichen Beigeschmack hat. Wird die Heavy-user-Forschung damit zum paranormalen Grenzbereich der Psychiatrie - sozusagen zur Paraversorgungsforschung? Kritiker sehen das wohl so.

Dennoch sind es nicht die definitorischen Unsicherheiten, die an der Heavy-user-Forschung primär kritisiert werden. Der am häufigsten zu hörende Vorwurf lässt sich in einem Wort zusammenfassen: Tautologie. Die Heavy-user-Forschung sei ein Zirkelschluss, heißt es, und man müsse sich nicht wundern, dass Patienten die Versorgungsangebote stark in Anspruch nehmen, heavy user sind, da man dies ja vorher so festgelegt habe.

Diese Kritik wäre berechtigt, wenn Patienten, die einmal heavy user sind, immer heavy user bleiben würden. Viele Untersuchungen sprechen aber dafür, dass dies nicht so sein muss. Zwar ist die frühere Intensität der Serviceinanspruchnahme ein wichtiges Indiz für die künftige Nutzung medizinischer Versorgung, aber es müssen weitere Aspekte hinzukommen, damit ein Patient, der letztes Jahr ein heavy user war, es auch im Folgejahr sein wird. Die Forschung versucht deshalb, Prädiktoren zu identifizieren, mit denen potenzielle heavy user möglichst am Anfang einer Phase überdurchschnittlich starker Versorgungsinanspruchnahme erkannt werden können.

Ein zweiter Vorwurf, der weniger oft zu hören, aber nicht weniger ernst zu nehmen ist, lautet: Stigmatisierung. Und in der Tat sind die Begriffe „heavy user“ oder „high utilizer“ im deutschen Sprachgebrauch eher negativ konnotiert. Sie repräsentieren die Perspektive der Kostenträger und implizieren, dass die so bezeichneten Patienten medizinische Versorgung öfter als nötig in Anspruch nehmen. Weniger stigmatisierend klingt dagegen die deutsche Übersetzung „Patienten mit starker Inanspruchnahme von Versorgungsleistungen“. Diese Bezeichnung ist jedoch auch weniger prägnant und nicht so etabliert wie die englischen Begriffe und wird daher in der Praxis eher als weiteres Synonym verwendet.

Die Tatsache, dass bestimmte Patienten medizinische Versorgungsangebote überdurchschnittlich häufig nutzen, muss aber nicht in jedem Fall einen negativen Beiklang haben. Aus der Perspektive der betroffenen Einrichtungen könnten heavy user durchaus auch positiv wahrgenommen werden. Immerhin sind sie nicht nur die eifrigsten Nutzer medizinischer Versorgung, sondern auch diejenigen Patienten, die durch ihr ständiges Wiederkommen das Behandlungsangebot und die Bemühungen des Personals auf ihre Weise besonders wertschätzen. Insofern würde es nicht verwundern, wenn die Leiter medizinischer Einrichtungen sich eine Einstellung zu Eigen machen würden, die Betriebswirtschaftler schon lange haben, nämlich: „Heavy user sind Kunden, die im Gegensatz zu light usern ein Produkt besonders häufig nachfragen und deshalb die Hauptzielgruppe des Marketing darstellen“ [5].

Aber ganz so einfach scheint die Sache im Gesundheitswesen doch nicht zu sein. Medizinische Versorgung zielt in erster Linie auf Heilung oder Rehabilitation - also auf eine Verbesserung des gesundheitlichen Status quo, die langfristig dazu führt, dass eine
Behandlung immer seltener benötigt wird. Bei heavy usern lässt sich ein solches Inanspruchnahmemuster jedoch nicht beobachten. Das behandelnde Personal hat deshalb oft das Gefühl, den betroffenen Patienten nicht richtig helfen zu können. Daher wird es nicht daran denken, die Versorgungsinanspruchnahme von heavy usern durch Marketing gezielt zu fördern, sondern eher resigniert als erfreut reagieren, wenn ein Patient zum zehnten Mal innerhalb eines Jahres zur stationären Aufnahme erscheint.

Starke Inanspruchnahme medizinischer Versorgung hat aber noch eine andere, tatsächlich positive Seite: die Fähigkeit der Patienten, einen Ausweg aus einer für sie unbefriedigenden Situation zu finden. So stellten Lewis u. Hugi im Ergebnis einer qualitativen Untersuchung fest: „The continued use of inpatient facilities reflects less the inadequacy of clinical services or the severe disability of clients that it does the purposeful behavior of resource-poor citizens who can avail themselves of these stations when they feel it is necessary. The hospital... is used by the chronically treated as a social resource, not a clinical resource“ ([6], S. 218).

Wenn man dieser Beobachtung glaubt, ist heavy use für die betroffenen Patienten alles andere als ein Problem. Aber warum wird das „Phänomen“ dann so hartnäckig untersucht? Bei dieser Frage wird immer wieder damit argumentiert, dass die häufige Inanspruchnahme stationärer Versorgung eine erfolgreiche Gemeindeintegration behindere und sich insofern nachteilig auf die betroffenen Patienten auswirke. Aber das ist nur die professionelle Sichtweise. Ob die Gemeindeintegration tatsächlich auch für heavy user ein vordringliches Ziel ist, wissen wir nicht. Und wir wissen ebenso wenig, wie sehr heavy user wirklich an ihrer starken Inanspruchnahme medizinischer Versorgung leiden.

Sehr wahrscheinlich ist heavy use eher ein Problem der Kostenträger und des behandelnden Personals, als ein Problem der betroffenen Patienten.

Demnach geht es in der Heavy-user-Forschung auch darum, alternative Versorgungsangebote zu konzipieren, die für die Patienten den gleichen Zweck erfüllen, wie die bislang stark in Anspruch genommenen Dienste, aber weniger kostenintensiv und weniger auf eine Einrichtung fixiert sind. Eine solche Möglichkeit bietet sich in erster Linie bei Patienten, die medizinische Versorgung häufig aufgrund sozialer Probleme nutzen. Dagegen sind Interventionen bei Patienten, die Behandlungsangebote primär aufgrund einer akuten Verschlechterung ihres psychischen Zustands in Anspruch nehmen, eher schwierig. Hier könnte nur mit besseren Therapiekonzepten reagiert werden, die bislang jedoch nicht zur Verfügung stehen.

Ob es tatsächlich heavy user gibt, die primär soziale Unterstützung benötigen und heavy user, die primär medizinische Probleme haben, muss noch untersucht werden. Da sich die Schwere der Grunderkrankung und das Ausmaß sozialer Probleme aber gegenseitig bedingen, kann man in der Praxis davon ausgehen, dass beide Faktoren eine Rolle bei der starken Inanspruchnahme medizinischer Versorgung spielen. Daher ist es sinnvoll, allen heavy usern alternative Versorgungsangebote zur Lösung sozialer Probleme zu unterbreiten. 
In mehreren Modellprogrammen konnte gezeigt werden, dass auf diese Weise nicht nur die starke Inanspruchnahme teurer Versorgungsangebote zurückgeht, sondern dass sich auch das soziale Funktionsniveau und die Zufriedenheit der Patienten verbessern [7 - 10]. Allerdings sind solche alternativen Versorgungsstrategien nur sinnvoll, wenn es darum geht, die starke Inanspruchnahme kostenintensiver Leistungen zu reduzieren. Das bedeutet, dass sich die Entwicklung und Evaluation geeigneter Interventionsprogramme in erster Linie auf heavy user stationärer Versorgung konzentriert.

Heavy usern medizinischer Dienste, die nicht so kostenintensiv sind, können in der Regel keine alternativen Versorgungsangebote unterbreitet werden. Hier ist das Personal der jeweiligen Einrichtungen gefordert, den Kontakt mit den heavy usern selbständig zu strukturieren und auf diese Weise sowohl für die Patienten, als auch für die Mitarbeiter befriedigend zu gestalten.

Bislang war die Heavy-user-Forschung eine Domäne angloamerikanischer Wissenschaftler. Seit einigen Jahren etablieren sich aber auch im deutschen Sprachraum zunehmend Arbeitsgruppen, die sich mit dem Problem starker Inanspruchnahme medizinischer Versorgung auseinandersetzen. In Deutschland wird dieses Forschungsgebiet seit dem Jahr 2000 im Rahmen eines Versorgungsforschungsprogramms gezielt durch das Bundesministerium für Bildung und Forschung sowie die Spitzenverbände der gesetzlichen Krankenkassen gefördert.

Bleibt zu hoffen, dass es mit dem hierzulande neuen Forschungsbereich nicht nur gelingt, die Untersuchungsansätze der anglo- amerikanischen Studien im deutschen Sprachraum zu replizieren, sondern einen darüber hinausgehenden, eigenständigen Beitrag zur Aufklärung des Heavy-user-Phänomens zu leisten. Offene Fragen gibt es genug.

\section{Literatur}

${ }^{1}$ Lucas B, Harrison-Read P, Tyrer P et al. Costs and characteristics of heavy inpatient service users in outer London. Int J Soc Psychiatry 2001; 47 (1): $63-74$

${ }^{2}$ Casper ES, Pastva G. Admission histories, patterns, and subgroups of the heavy users of a state psychiatric hospital. Psychiatr Q 1990; 61: $121-134$

${ }^{3}$ Casper ES, Donaldson MA. Subgroups in the population of frequent users of inpatient services. Hosp Community Psychiatry 1990; 41 (2): $189-191$

${ }^{4}$ Casper ES, Regan JR. Reasons for admission among six profile subgroups of recidivists of inpatient services. Can J Psychiatry 1993; 38: $657-661$

${ }^{5}$ Customer Relationship Management - Glossar. http://www.wolbersen-associates.de/crm/glossar_h.html

${ }^{6}$ Lewis PA, Hugi R. Therapeutic stations and the chronically treated mentally ill. Soc Serv Rev 1981; 55: 206-220

${ }^{7}$ Witheridge TF, Dincin J, Appleby L. Working with the most frequent recidivists: a total team approach to assertive resource management. Psychosocial Rehabilitation Journal 1982; 5: 9-11

8 Bond GR, Miller LD, Krumwied MHA et al. Assertive case management in three CMHCs: a controlled study. Hosp Community Psychiatry 1988; 35 : $411-417$

${ }^{9}$ Dharwadkar N. Effectiveness of an assertive outreach community treatment program. Aust N Z J Psychiatry 1994; 28: 244-249

${ }^{10}$ Quinlivan R, Hough R, Crowell A et al. Service utilization and costs of care for severely mentally ill clients in an intensive case management program. Psychiatr Serv 1995; 46: 365 - 371 